\title{
Search for $C P$ violation and rare decays in charm sector at Belle
}

\section{Minakshi Nayak*i}

Wayne state University USA, High Energy Accelerator Research Organization (KEK)

E-mail: minakshi.nayak@wayne.edu

\begin{abstract}
Measurement of branching fractions, and $C P$ asymmetries in $D^{0}$ decays are interesting as any difference with respect to the Standard Model prediction would be an indication of new physics. Using the full data sample collected with the Belle detector located at the KEKB asymmetricenergy $e^{+} e^{-}$(3.5 on $8 \mathrm{GeV}$ ) collider, we present the results of the first measurement of $T$-odd moments in the decay $D^{0} \rightarrow K_{S} \pi^{+} \pi^{-} \pi^{0}$; measurement of branching fractions and $C P$ asymmetries in $D^{0} \rightarrow V \gamma\left(V=\phi, K^{* 0}, \rho^{0}\right), K_{S}^{0} K_{S}^{0}$ decays; and the first search for $D^{0}$ decays to invisible final states.
\end{abstract}

EPS-HEP 2017, European Physical Society conference on High Energy Physics 5-12 July 2017

Venice, Italy

* Speaker.

${ }^{\dagger}$ On behalf of the Belle Collaboration 


\section{Introduction}

In the Standard Model (SM), Charge-Parity $(C P)$ violation in charm meson decays is predicted to be very small $\left[\mathscr{O}\left(10^{-3}\right)\right]$. Any enhancement with respect to the SM prediction can be due to new particles or new interactions which are not included in the SM [1]. Here, we present the results of the first measurement of the $T$-odd moment asymmetry in the decay $D^{0} \rightarrow K_{S} \pi^{+} \pi^{-} \pi^{0}$; the branching fractions and $C P$ asymmetries in $D^{0} \rightarrow V \gamma\left(V=\phi, K^{* 0}, \rho^{0}\right), D^{0} \rightarrow K_{S}^{0} K_{S}^{0}$ decays; and the first search for $D^{0}$ decays to invisible final states using the full Belle data set which corresponds to an integrated luminosity of around $1 \mathrm{ab}^{-1}$.

\section{First measurement of the $T$-odd moments in the decay $D^{0} \rightarrow K_{S} \pi^{+} \pi^{-} \pi^{0}$}

The self conjugate decay to the final state $D^{0} \rightarrow K_{S} \pi^{+} \pi^{-} \pi^{0}$ has a large branching fraction of $5.2 \%$ [2] which allows for a precise test of $C P$ symmetry as a sample of $\mathscr{O}\left(10^{6}\right)$ decays is expected. Previously the decay was studied by the MARK III Collaboration with a sample of only 140 events which corresponds to a data sample of $9.56 \mathrm{pb}^{-1}$ [3]. Here, we present the first measurement of the time-reversal $(T)$ asymmetry in $D^{0} \rightarrow K_{S} \pi^{+} \pi^{-} \pi^{0}$ decays which has two neutral particles in the final state. The measurement is sensitive to $C P$ violation via the $C P T$ theorem. The measurement is performed via the scalar triple product: $C_{T}=\mathbf{p}_{\mathbf{K}_{\mathbf{S}}} \cdot\left(\mathbf{p}_{\pi^{+}} \times \mathbf{p}_{\pi^{-}}\right)$, where $\mathbf{p}_{\mathbf{K}_{\mathbf{S}}}, \mathbf{p}_{\pi^{+}}$, and $\mathbf{p}_{\pi^{-}}$are the momenta of the $D^{0}$ daughters $K_{S}, \pi^{+}, \pi^{-}$respectively. Similarly, $\bar{C}_{T}$ can be defined for the $\bar{D}^{0}$ daughter particles. The two asymmetry parameters for $D^{0}$ and $\bar{D}^{0}$ are defined as

$$
A_{T}=\frac{\Gamma\left(C_{T}>0\right)-\Gamma\left(C_{T}<0\right)}{\Gamma\left(C_{T}>0\right)+\Gamma\left(C_{T}<0\right)}, \bar{A}_{T}=\frac{\Gamma\left(-\bar{C}_{T}>0\right)-\Gamma\left(-\bar{C}_{T}<0\right)}{\Gamma\left(-\bar{C}_{T}>0\right)+\Gamma\left(-\bar{C}_{T}<0\right)}
$$

Here $\Gamma$ is the partial decay rate. The above asymmetries can be nonzero due to the final state interaction (FSI) effects [4], but these effects are eliminated by taking the difference between $A_{T}$ and $\bar{A}_{T}$ as a $C P$ violation sensitive parameter which is defined as

$$
a_{C P}^{T-\text { odd }}=\frac{1}{2}\left(A_{T}-\bar{A}_{T}\right) .
$$

\subsection{Signal extraction}

By using the analysis technique as described in [5], $D^{* \pm}$ and $D^{0}$ mesons are reconstructed from the decays: $D^{* \pm} \rightarrow D^{0} \pi_{\text {slow }}^{ \pm}, D^{0} \rightarrow K_{S} \pi^{+} \pi^{-} \pi^{0}$. As the final state is the same for both $D^{0}$ and $\bar{D}^{0}$, so we use the charge of $\pi_{\text {slow }}^{ \pm}$to distinguish the flavour of the $D$ meson. The $\pi_{\text {slow }}^{ \pm}$is so called because it carries a small momentum compared to the $D^{0}$ meson. We extract the signal yield by performing a two-dimensional unbinned maximum likelihood fit to the mass difference $\Delta M\left(\mathrm{M}_{\mathrm{D}^{* \pm}}-\mathrm{M}_{\mathrm{D}^{0}}\right)$ and $\mathrm{M}_{\mathrm{D}^{0}}$, where $\mathrm{M}_{\mathrm{D}^{* \pm}}$ and $\mathrm{M}_{\mathrm{D}^{0}}$ are the masses of $D^{* \pm}$ and $D^{0}$ mesons respectively. The fit results are shown in Figure 1. The total signal yield extracted from the above fit is 744,509 $\pm 1,622$ events.

\subsection{Results}

The asymmetry obtained for the decay is $A_{T}=(11.60 \pm 0.19) \%$, the large value of which is due to the FSI effects. The $T$-odd moment asymmetry is $a_{C P}^{T-\text { odd }}=\left(-0.28 \pm 1.38_{-0.76}^{+0.23}\right) \times 10^{-3}$ 

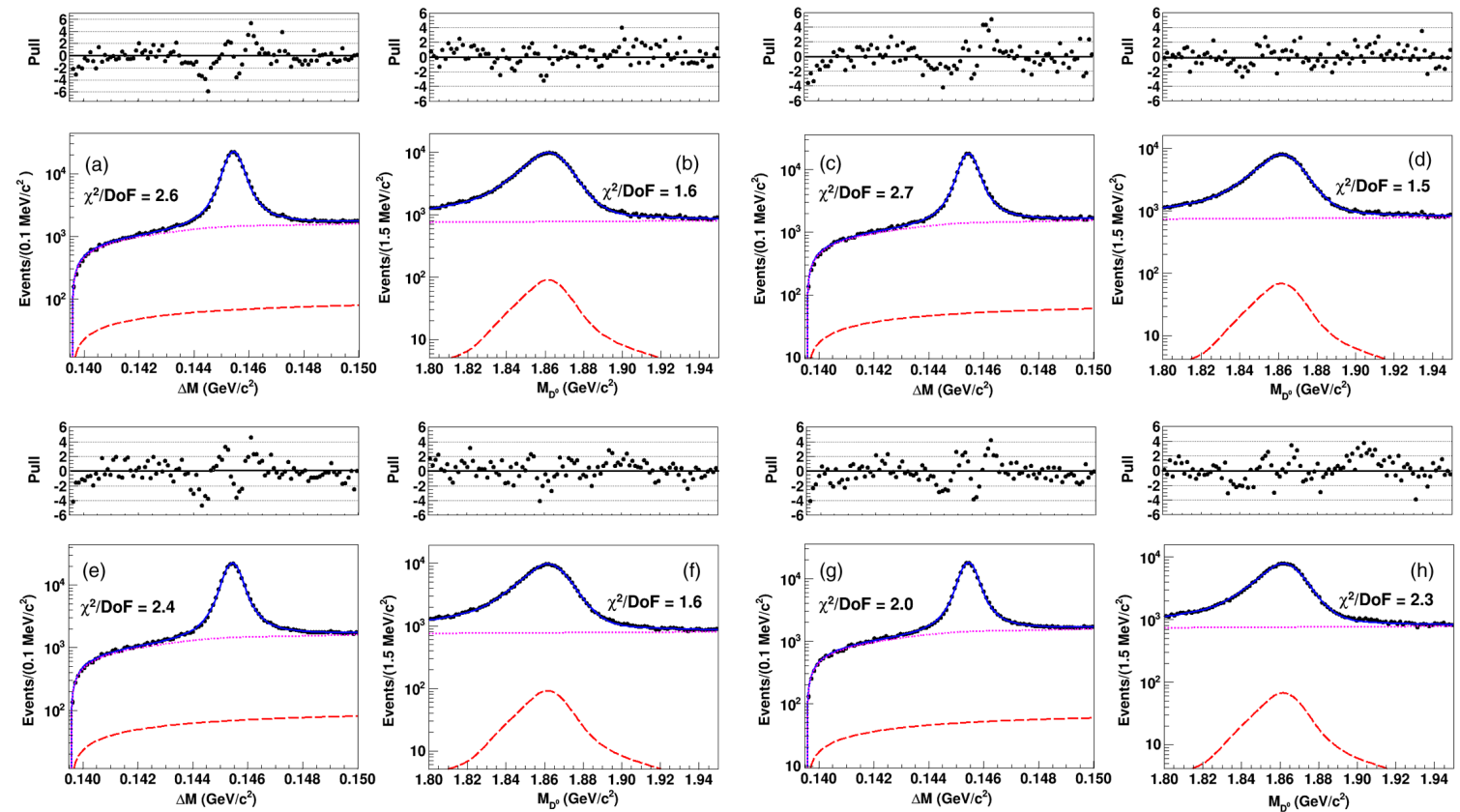

Figure 1: [colour online]. The signal-enhanced logarithmic distributions of (a) $\Delta M$ and (b) $M_{D^{0}}$ for $D^{0}$ with $C_{T}>0$, (c) $\Delta M$ and (d) $M_{D^{0}}$ for $D^{0}$ with $C_{T}<0$, (e) $\Delta M$ and (f) $M_{D^{0}}$ for $\bar{D}^{0}$ with $-\bar{C}_{T}>0$, (g) $\Delta M$ and (h) $M_{D^{0}}$ ) for $\bar{D}^{0}$ with $-\bar{C}_{T}<0$. In these plots, points with error bars represent data, while the total best-fit projections are shown by the solid blue curves, for which the combinatorial background component is shown by the dotted magenta curves and the random $\pi_{\text {slow }}^{ \pm}$background is shown by the dashed red curves. The normalised residuals (pulls) are shown above each plot.

which is consistent with no $C P$ violation [5]. The $K_{S} \pi^{+} \pi^{-} \pi^{0}$ phase space has been divided in to nine bins depending upon the intermediate resonance contributions as listed in Table 1. The results of $a_{C P}^{T-\text { odd }}$ in all bins are also consistent with no $C P$ violation, but $A_{T}$ can vary significantly due to different resonance contributions. The result constitutes one of the most precise tests of $C P$ violation in the $D$ meson system, but it is still statistically dominated, and hence the sensitivity can be improved by the upcoming Belle II experiment [6].

\section{Search for $C P$ asymmetry in $D^{0} \rightarrow V \gamma\left(V=\phi, \bar{K}^{* 0}, \rho^{0}\right)$ decays}

Radiative charm decays are dominated by long range non-perturbative processes that can enhance the branching fractions up to $\mathscr{O}\left(10^{-4}\right)$ from $\mathscr{O}\left(10^{-8}\right)$ which is obtained from short range processes. Again measurement of branching fractions can be used to test QCD based theoretical calculations of long-distance dynamics. Further, radiative charm decays are sensitive to new physics (NP) via $C P$ asymmetry $\left(\mathscr{A}_{C P}\right)$. Theoretical calculations $[7,8]$ predict that in SM extensions chromomagnetic dipole operators can raise the value of $\mathscr{A}_{C P}$ up to several percent for $D^{0} \rightarrow \phi \gamma$ and $D^{0} \rightarrow \rho^{0} \gamma$ decays. The decay $D^{0} \rightarrow \phi \gamma$ has been observed by Belle [9] using a data set of $78.1 \mathrm{fb}^{-1}$. Later with a $387.1 \mathrm{fb}^{-1}$ data sample the BaBar collaboration [10] measured branching fractions for both the decays $D^{0} \rightarrow \phi \gamma$ and $D^{0} \rightarrow \bar{K}^{* 0} \gamma$. The decay $D^{0} \rightarrow \rho^{0} \gamma$ had not been observed before, but with a $4.8 \mathrm{fb}^{-1}$ data set CLEO II [11] had placed an upper limit of $\mathscr{B}\left(D^{0} \rightarrow \rho^{0} \gamma\right)<2.4 \times 10^{-4}$. No $\mathscr{A}_{C P}$ measurements had been performed in $D^{0} \rightarrow V \gamma$ decays. 
Table 1: $A_{T}$ and $a_{C P}^{T \text {-odd }}$ values from different regions of $D^{0} \rightarrow K_{S}^{0} \pi^{+} \pi^{-} \pi^{0}$ phase space.

\begin{tabular}{llccr}
\hline \hline Bin & Resonance & $\begin{array}{c}\text { Invariant mass } \\
\text { requirement }\left(\mathrm{MeV} / c^{2}\right)\end{array}$ & $A_{T}\left(\times 10^{-2}\right)$ & $a_{C P}^{T-\text { odd }}\left(\times 10^{-3}\right)$ \\
\hline 1 & $K_{S}^{0} \eta$ & $M_{\pi^{+} \pi^{-} \pi^{0}}<590$ & $0.2 \pm 1.3 \pm 0.4$ & $4.6 \pm 9.5 \pm 0.2$ \\
2 & $K_{S}^{0} \omega$ & $762<M_{\pi^{+} \pi^{-}} \pi^{0}<02$ & $3.6 \pm 0.5 \pm 0.5$ & $-1.7 \pm 3.2 \pm 0.7$ \\
3 & $K^{*-} \rho^{+}$ & $790<M_{K_{S}^{0} \pi^{-}}<994$ & $6.9 \pm 0.3_{-0.5}^{+0.6}$ & $0.0 \pm 2.0_{-1.4}^{+1.6}$ \\
& & $610<M_{\pi^{+}} \pi^{0}<960$ & & \\
4 & $K^{*+} \rho^{-}$ & $790<M_{K_{S}^{0} \pi^{+}}<994$ & $22.0 \pm 0.6 \pm 0.6$ & $1.2 \pm 4.4_{-0.4}^{+0.3}$ \\
& & $610<M_{\pi^{-}} \pi^{0}<960$ & & \\
5 & $K^{*-} \pi^{+} \pi^{0}$ & $790<M_{K_{S}^{0} \pi^{-}}<994$ & $25.5 \pm 0.7 \pm 0.5$ & $-7.1 \pm 5.2_{-1.3}^{+1.2}$ \\
6 & $K^{*+} \pi^{-} \pi^{0}$ & $790<M_{K_{S}^{0} \pi^{+}}<994$ & $24.5 \pm 1.0_{-0.6}^{+0.7}$ & $-3.9 \pm 7.3_{-1.2}^{+2.4}$ \\
7 & $K^{* 0} \pi^{+} \pi^{-}$ & $790<M_{K_{S}^{0} \pi^{0}}<994$ & $19.7 \pm 0.8_{-0.5}^{+0.4}$ & $0.0 \pm 5.6_{-0.9}^{+1.1}$ \\
8 & $K_{S}^{0} \rho^{+} \pi^{-}$ & $610<M_{\pi^{+} \pi^{0}}<960$ & $13.2 \pm 0.9 \pm 0.4$ & $7.6 \pm 6.1_{-0.0}^{+0.2}$ \\
9 & Remainder & - & $20.5 \pm 1.0_{-0.6}^{+0.5}$ & $1.8 \pm 7.4_{-5.3}^{+2.1}$ \\
\hline \hline
\end{tabular}

\subsection{Analysis overview}

We measure branching fractions and $C P$ asymmetries for the decay modes: $D^{0} \rightarrow \phi\left(K^{+} K^{-}\right) \gamma$, $D^{0} \rightarrow \rho^{0}\left(\pi^{+} \pi^{-}\right) \gamma$, and $D^{0} \rightarrow \bar{K}^{* 0}\left(K^{-} \pi^{+}\right) \gamma$ relative to the normalisation modes: $D^{0} \rightarrow K^{+} K^{-}$, $D^{0} \rightarrow \pi^{+} \pi^{-}$and $D^{0} \rightarrow K^{-} \pi^{+}$respectively. To cancel several sources of systematic uncertainties that are common to both the signal and normalisation modes, we choose the normalisation modes that decay to the same charged final state particles as the signal decays. $D^{0}$ mesons are reconstructed from the decay $D^{* \pm} \rightarrow D^{0} \pi_{\text {slow }}^{ \pm}$in order to identify the flavour of the $D^{0}$ meson and to suppress combinatorial background.

The signal branching fraction $\mathscr{B}_{\text {sig }}$ is defined as

$$
\mathscr{B}_{\text {sig }}=\mathscr{B}_{\text {norm }} \times \frac{N_{\text {sig }}}{N_{\text {norm }}} \times \frac{\varepsilon_{\text {norm }}}{\varepsilon_{\text {sig }}} .
$$

Here, $N$ is the extracted signal yield, $\varepsilon$ the reconstructed efficiency, and $\mathscr{B}$ is the branching fraction.

The measured raw asymmetry is

$$
\mathscr{A}_{\text {raw }}=\frac{N\left(D^{0} \rightarrow f\right)-N\left(\bar{D}^{0} \rightarrow \bar{f}\right)}{N\left(D^{0} \rightarrow f\right)+N\left(\bar{D}^{0} \rightarrow \bar{f}\right)}=\mathscr{A}_{C P}+\mathscr{A}_{F B}+\mathscr{A}_{\varepsilon}^{ \pm},
$$

which is the sum of the physical $C P$ asymmetry $\left(\mathscr{A}_{C P}\right)$, the forward-backward production asymmetry $\left(\mathscr{A}_{F B}\right)$, and the asymmetry due to different detection efficiencies for positively and negatively charged particles $\left(\mathscr{A}_{\varepsilon}^{ \pm}\right) . \mathscr{A}_{F B}$ and $\mathscr{A}_{\varepsilon}^{ \pm}$are eliminated by comparing with the normalisation mode. Hence the $\mathscr{A}_{C P}$ asymmetry of the signal mode can be expressed as

$$
\mathscr{A}_{C P}^{\text {sig }}=\mathscr{A}_{\text {raw }}^{\text {sig }}-\mathscr{A}_{\text {raw }}^{\text {norm }}+\mathscr{A}_{C P}^{\text {norm }}
$$

$\mathscr{A}_{C P}^{\text {norm }}$ is the world average $C P$ asymmetry of the normalisation mode [2].

\subsection{Signal extraction}

By using the analysis technique as described in [12], signal events are extracted via a simultaneous unbinned maximum likelihood fit to the mass of $D^{0}$ meson $M\left(D^{0}\right)$ and cosine of the helicity 
angle $\cos \left(\theta_{H}\right)$, where $\cos \left(\theta_{H}\right)$ is the angle between the $D^{0}$ and a daughter particle of the $V$ meson in the rest frame of the $V$ meson.

The dominant backgrounds arise from the decays $D^{0} \rightarrow h^{+} h^{-} \pi^{0}(h=K, \pi)$, with the $\pi^{0}$ decaying to a pair of photons. If one of the daughter photons is missed in the reconstruction, the final state mimics the signal. Such events are suppressed by using a dedicated $\pi^{0}$ veto which uses an artificial neural network. The new veto rejects about $60 \%$ of the background (and rejects $13 \%$ more background than the previous veto used in the Belle analysis) while retaining $85 \%$ of the signal.

The fit results are shown in Figure 2 for all three signal modes. The extracted signal yields are $500 \pm 85$ ( $\rho^{0} \gamma$ mode), $9104 \pm 396\left(\bar{K}^{* 0} \gamma\right.$ mode), $524 \pm 35$ events ( $\phi \gamma$ mode).
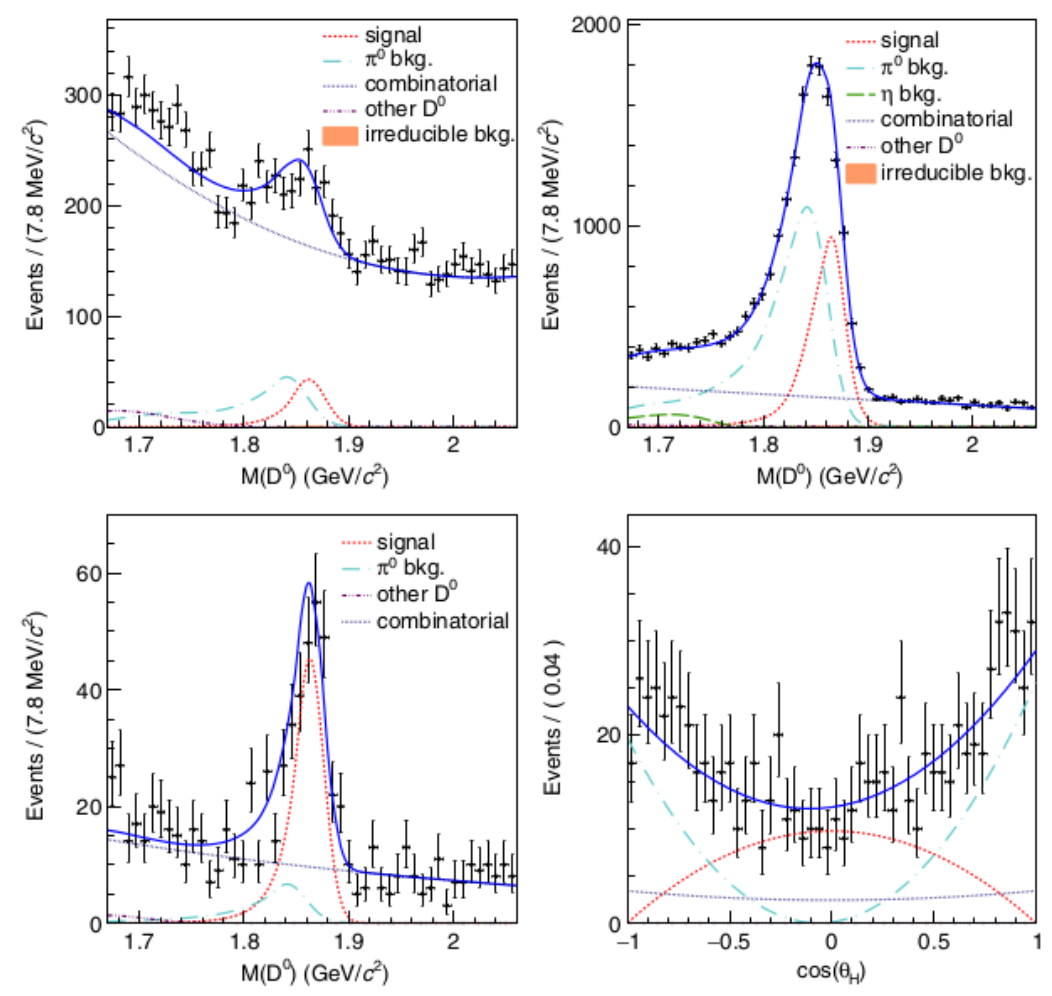

Figure 2: [colour online]. The signal enhanced plot of the combined $M\left(D^{0}\right)$ distributions for $D^{0} \rightarrow \rho^{0} \gamma$ (top left) and $D^{0} \rightarrow \bar{K}^{* 0} \gamma$ (top right). The bottom plots are the signal enhanced $M\left(D^{0}\right)$ (left) and $\cos \left(\theta_{H}\right)$ (right) distributions for $D^{0} \rightarrow \phi \gamma$. In these plots, points with error bars represent data while the total best-fit projection is shown with the solid blue curve, with the fit components identified in the panel legend.

\subsection{Results}

The obtained branching fractions are

$$
\begin{aligned}
\mathscr{B}\left(D^{0} \rightarrow \rho^{0} \gamma\right) & =(1.77 \pm 0.30 \pm 0.07) \times 10^{-5} \\
\mathscr{B}\left(D^{0} \rightarrow \phi \gamma\right) & =(2.76 \pm 0.19 \pm 0.10) \times 10^{-5} \\
\mathscr{B}\left(D^{0} \rightarrow \bar{K}^{* 0} \gamma\right) & =(4.66 \pm 0.21 \pm 0.21) \times 10^{-4}
\end{aligned}
$$

where the first uncertainty is statistical and the second is systematic. For the $\rho^{0}$ mode we report the first observation of the decay. The result for the $\phi$ mode is an improvement on the previous Belle 
result but is consistent with the world average [2], and the result of $\bar{K}^{* 0}$ mode is $3.3 \sigma$ above the result of the BaBar analysis.

We also report the first measurement of $\mathscr{A}_{C P}$ in the decays $D^{0} \rightarrow V \gamma$. The results are

$$
\begin{aligned}
\mathscr{A}_{C P}\left(D^{0} \rightarrow \rho^{0} \gamma\right) & =+(0.056 \pm 0.152 \pm 0.006), \\
\mathscr{A}_{C P}\left(D^{0} \rightarrow \phi \gamma\right) & =-(0.094 \pm 0.066 \pm 0.001), \\
\mathscr{A}_{C P}\left(D^{0} \rightarrow \bar{K}^{* 0} \gamma\right) & =-(0.003 \pm 0.020 \pm 0.000),
\end{aligned}
$$

and are consistent with no $C P$ violation, but the statistical uncertainty is dominant, and hence the sensitivity can be greatly enhanced by the Belle II experiment [6].

\section{Measurement of $C P$ asymmetry in $D^{0} \rightarrow K_{S}^{0} K_{S}^{0}$ decay}

Singly Cabibbo-suppressed decays like $D^{0} \rightarrow K_{S}^{0} K_{S}^{0}$ are interesting as the possibility of interference with NP amplitudes could lead to large nonzero $C P$ violation. A recent SM based calculation obtains an upper limit of $1.1 \%$ for direct $C P$ violation in this decay [13]. The previous search for this decay was first performed by CLEO [14] using a data sample of $13.7 \mathrm{fb}^{-1}$ and measured a $C P$ asymmetry of $(-23 \pm 19) \%$. Later LHCb measured the $C P$ asymmetry as $(-2.9 \pm 5.2 \pm 2.2) \%$ [15]. Both the results are consistent with no $C P$ violation. Recently BESIII, using a data sample of $2.93 \mathrm{fb}^{-1}$, reported a branching fraction of $(1.67 \pm 0.11 \pm 0.11) \times 10^{-4}$ [16] for this mode. Belle significantly improved these measurements using a data sample of $921 \mathrm{fb}^{-1}$.

\subsection{Analysis overview}

We measure branching fractions and $C P$ asymmetries for the decay $D^{0} \rightarrow K_{S}^{0} K_{S}^{0}$, relative to the normalisation mode $D^{0} \rightarrow K_{S}^{0} \pi^{0}$. Similarly to the other two analyses described above, $D^{0}$ mesons are reconstructed from the decay $D^{* \pm} \rightarrow D^{0} \pi_{\text {slow }}^{ \pm}$in order to identify the flavour of the $D^{0}$ meson and to suppress combinatorial background.

The measured raw asymmetry is

$$
\mathscr{A}_{\text {raw }}=\frac{N\left(D^{0} \rightarrow f\right)-N\left(\bar{D}^{0} \rightarrow \bar{f}\right)}{N\left(D^{0} \rightarrow f\right)+N\left(\bar{D}^{0} \rightarrow \bar{f}\right)}=\mathscr{A}_{C P}+\mathscr{A}_{F B}+\mathscr{A}_{\varepsilon}^{ \pm}+\mathscr{A}_{\varepsilon}^{K},
$$

and the $\mathscr{A}_{C P}$ asymmetry of the signal mode can be expressed as

$$
\mathscr{A}_{C P}^{\text {sig }}=\mathscr{A}_{\text {raw }}^{\text {sig }}-\mathscr{A}_{\text {raw }}^{\text {norm }}+\mathscr{A}_{C P}^{\text {norm }}++\mathscr{A}_{\varepsilon}^{K},
$$

where $\mathscr{A}_{\varepsilon}^{K}=(-0.11 \pm 0.01) \%$ [17] is the asymmetry originating from the different strong interactions of $K^{0}$ and $\bar{K}^{0}$ mesons with detector material.

\subsection{Signal extraction}

By using the analysis technique as described in [18], signal is extracted via a simultaneous unbinned maximum likelihood fit of the $\Delta M$ distributions for $D^{*+}$ and $D^{*-}$. The fit results are shown in Figure 3. The extracted signal yields are $4755 \pm 79\left(K_{S}^{0} K_{S}^{0}\right.$ mode) and $475439 \pm 767$ events ( $K_{S}^{0} \pi^{0}$ normalisation mode). 

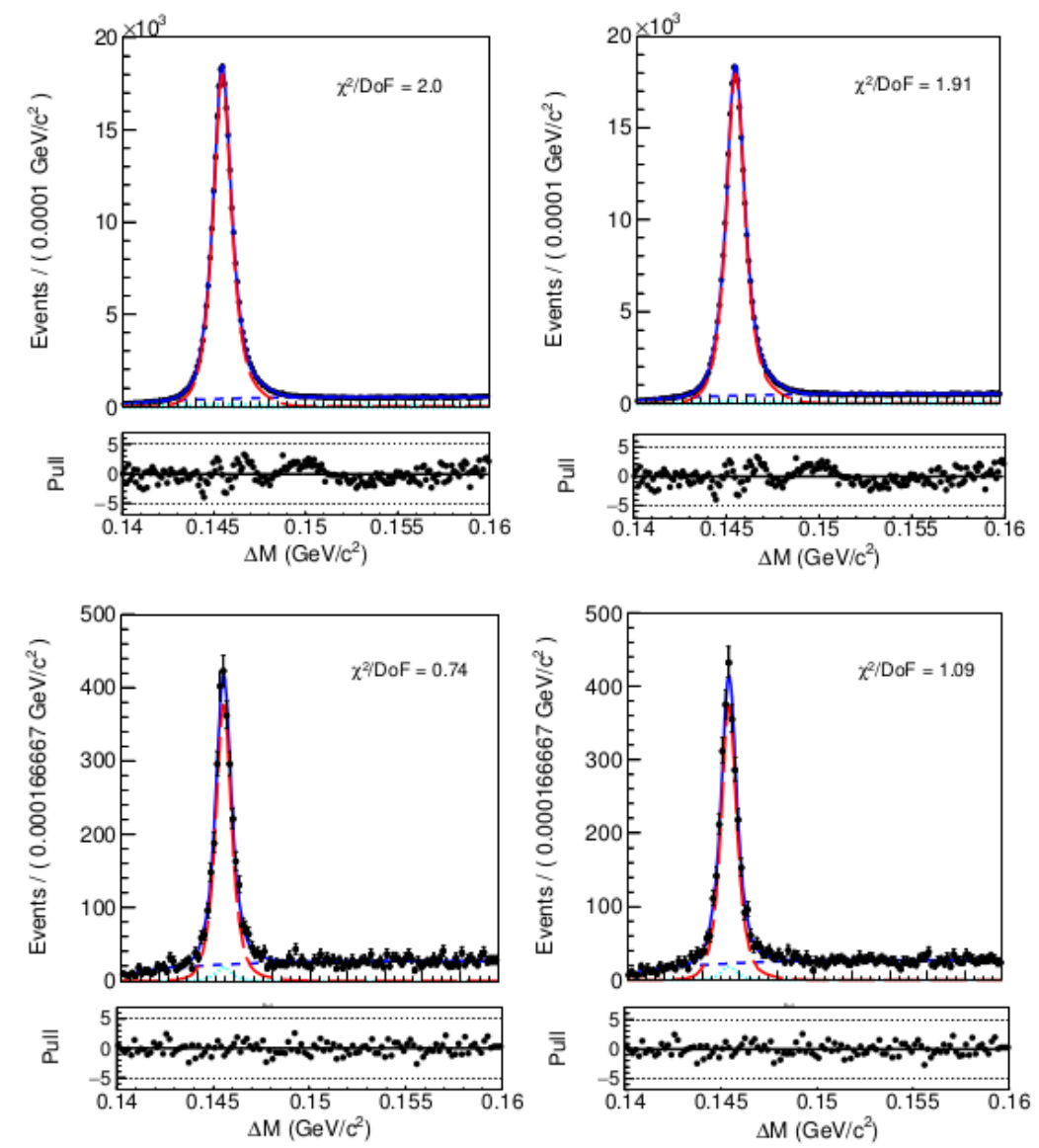

Figure 3: [colour online]. Distributions of the mass difference $\Delta M$ for selected $D^{*+}$ (left) and $D^{*-}$ (right) candidates, reconstructed from $D^{0} \rightarrow K_{S}^{0} \pi^{0}$ (top) and $D^{0} \rightarrow K_{S}^{0} K_{S}^{0}$ (bottom). In these plots, points with error bars represent data while the total best-fit projection is shown with the solid blue curve, with the signal component (long-dashed red curve), the peaking background component (dotted cyan), and the combinatorial background component (dashed blue) shown. The normalised residuals (pulls) are shown below each plot.

\subsection{Result}

The results for the branching fraction and $C P$ asymmetry are

$$
\begin{gathered}
\mathscr{B}\left(D^{0} \rightarrow K_{S}^{0} K_{S}^{0}\right)=(1.32 \pm 0.02 \pm 0.04 \pm 0.04) \times 10^{-4}, \\
\mathscr{A}_{C P}\left(D^{0} \rightarrow K_{S}^{0} K_{S}^{0}\right)=-(0.02 \pm 1.53 \pm 0.02 \pm 0.17) .
\end{gathered}
$$

where the first uncertainty is statistical, the second is the systematic, and the third is due to the uncertainty on $A_{C P}(\mathscr{B})$ of $D^{0} \rightarrow K_{S}^{0} \pi^{0}$. The result of $\mathscr{A}_{C P}$ is consistent with no $C P$ violation and represents a significant improvement over the previous measurements. The branching fraction is consistent with the world average and $2.3 \sigma$ away from the recent BESIII measurement. Both results are the most precise measurements made for the $D^{0} \rightarrow K_{S}^{0} K_{S}^{0}$ decay.

\section{First search for $D^{0}$ decays to invisible final states}

By using the analysis technique as described in [19], the signal yield is extracted from a two- 
dimensional extended unbinned maximum likelihood fit to $M_{D^{0}}$ and the residual energy in the ECL $E_{E C L}$. The projections of the fit are shown in Figure 4. The extracted signal yield is $-6.3_{-21.0}^{+22.5}$ events, which is consistent with zero.
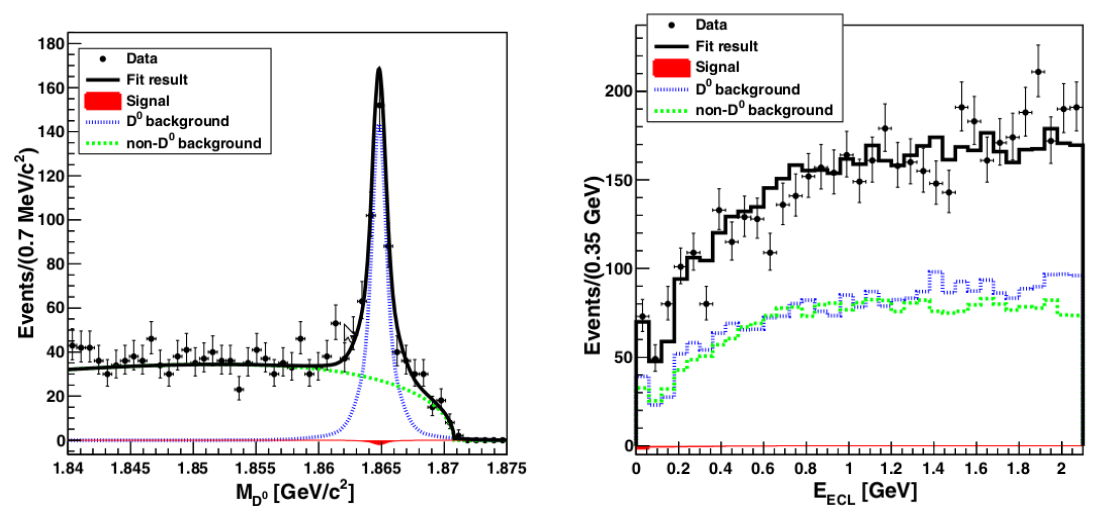

Figure 4: [colour online]. In these plots, points with error bars represent data while the total fit projection is shown with the solid black curve, with the fit components identified in the panel legend.

The upper limit on the branching fraction obtained for this decay is $9.4 \times 10^{-5}$ at the $90 \%$ confidence level.

\section{References}

[1] Y. Grossman, A. L. Kagan, and Y. Nir, Phys. Rev. D 75, 036008 (2007).

[2] C. Patrignani et al. (Particle Data Group Collaboration), Chin. Phys. C 40, 100001 (2016).

[3] D. Coffman et al. (MARK III Collaboration), Phys. Rev. D 45, 2196 (1992).

[4] I. I. Bigi, arXiv:hep-ph/0107102.

[5] K. Prasanth et al. (Belle Collaboration), Phys. Rev. D 95, 091101 (R) (2017).

[6] T. Abe et al. (Belle II Collaboration), arXiv:1011.0352.

[7] G. Isidori and J. F. Kamenik, Phys. Rev. Lett. 109, 171801 (2012).

[8] J. Lyon and R. Zwicky, arXiv:1210.6546.

[9] O. Tajima et al. (Belle Collaboration), Phys. Rev. Lett. 92, 101803 (2004).

[10] B. Aubert et al. (BABAR Collaboration), Phys. Rev. D 78, 071101(R) (2008).

[11] D. M. Asner et al. (CLEO Collaboration), Phys. Rev. D 58, 092001 (1998).

[12] T. Nanut et al. (Belle Collaboration), Physical Review Letters 118, 051801 (2017).

[13] U. Nierste and A. Schacht, Phys. Rev. D 92, 054036 (2015).

[14] G. Bonvicini et al. (CLEO Collaboration), Phys. Rev. D 63071101 (R) (2001).

[15] R. Aaij et al. (LHCb Collaboration), JHEP 10, 055 (2015).

[16] M. Ablikim et al., (BESIII Collaboration), Phys. Lett. B 765231 (2017).

[17] B.R. Ko, E. Won, B. Golob and P. Pakhlov, Phys. Rev.D 84111501 (2011).

[18] N. Dash et al., (Belle Collaboration), arXiv:1705.05966.

[19] Y.-T. Lai et al., (Belle Collaboration), Phys. Rev. D 95, 011102 (R) (2017). 